\title{
Using Syndromic Surveillance Data to Aid Public Health Actions in Tennessee
}

\author{
Caleb Wiedeman ${ }^{1}$, Kevin Morris ${ }^{1}$, Cassandra Jones ${ }^{1}$, Marisa Hopper Hopper ${ }^{2}$, Paul E. \\ Petersen 1 \\ ${ }^{1}$ Tennessee Department of Health, Nashville, Tennessee, United States, ${ }^{2}$ Shelby County Health Department, Memphis, Tennessee, United \\ States
}

\section{Objective}

To demonstrate the utility of syndromic surveillance data in aiding public health actions and response across multiple investigations in Tennessee.

\section{Introduction}

Syndromic surveillance data is typically used for the monitoring of symptom combinations in patient chief complaints (i.e. syndromes) or health indicators within a population to inform public health actions. The Tennessee Department of Health collects emergency department (ED) data from more than 80 hospitals across Tennessee to support statewide situational awareness. Most hospitals in Tennessee provide data within 48 hours of the patient being seen in the emergency department.

The timeliness of syndromic surveillance data allow for rapid estimates of impact in emergency department populations. Tennessee has successfully used these data to monitor influenza, heat related illnesses, and emergency department impacts from disaster evacuations. In addition to assessing impact and trends, syndromic surveillance can also provide early warnings for conditions of public health concern and increase the lead time public health has to initiate a response.

In Tennessee, routine syndromic surveillance for mumps, hepatitis A, and other conditions has been successfully conducted statewide. Three successes from these surveillance efforts include detecting a clinically diagnosed but unreported case of mumps, early identification of hepatitis A cases during Tennessee's ongoing 2018 hepatitis A outbreak, and the detection of an epidemiologically unlikely clinical diagnosis of mumps associated with an exposure at a recreational center.

\section{Methods}

Syndromic surveillance data in Tennessee are monitored daily for chief complaints and discharge diagnosis codes that could require immediate public health actions. Chief complaints are monitored using ESSENCE's built in Record of Interest (ROI) syndrome while ICD10 and SNOMED codes are monitored for specific conditions of interest. Examples include mumps, measles, rubella, hepatitis A, and other immediately notifiable conditions that have time sensitive public health interventions associated with them.

\section{Results}

In 2017, Tennessee was investigating a large increase in mumps cases and outbreaks and exposure responses were occurring across the state. In early June, a patient was seen at a hospital emergency department with a chief complaint of right sided testicular swelling, right sided jaw swelling, and a measured temperature of 99.0 at the time of the visit. Based on presentation alone, the patient was diagnosed by the physician with uncomplicated mumps and discharged. Two days, but less than 48 hours after patient was seen at the emergency department, the discharge diagnosis of mumps was received in Tennessee's syndromic surveillance system and detected during routine review of incoming discharge diagnoses.

Upon detection in Tennessee's syndromic surveillance data, both the regional health department where the patient listed his residence and the regional health department where the hospital was located were notified of the ED visit. The hospital was immediately contacted that morning and further investigation revealed that the physician who diagnosed the patient with mumps never ordered laboratory testing to support the diagnosis and that the patient had remained in the jurisdictional area surrounding the hospital. Because no laboratory testing was ordered, the infection preventionist at the hospital was not made aware of the patient's diagnosis and no notification to public health had been made. 
Rapid contact and coordination with the hospital, regional health departments, and county health department allowed for the patient to be seen at a county health department for specimen collection on the same day the visit was detected. The patient was confirmed PCR positive for mumps the following week. Epidemiologic follow up with the patient revealed that the patient was not linked to any of the ongoing outbreaks in Tennessee, but had likely been exposed and exposed others at multiple out of state events. Tennessee was able to follow up with the affected states and notify them of the potential exposures.

In early 2018, Tennessee's ongoing hepatitis A outbreak was detected. To supplement traditional investigative efforts, monitoring for chief complaints generally indicative of hepatitis was initiated across the state, along with diagnosis code monitoring for hepatitis A and unspecified hepatitis. Although all ages were monitored, follow up was focused on visits occurring in the most prevalent age group $(18-44)$, chief complaints including substance abuse indicators, and ICD10 codes indicative of Hepatitis A.

Routine syndromic surveillance for hepatitis A identified visits meeting the outbreak characteristics which were referred to local and regional health departments for follow up. Although many visits were confirmed to have been already reported or ruled out (particularly in non-outbreak counties), there were numerous times when laboratory tested hepatitis A cases where detected in syndromic surveillance data prior to them being reported. Early detection provided by syndromic surveillance increased the lead time for public health to identify potential high risk contacts and initiate post-exposure prophylaxis.

In the summer of 2018, a young child with chief complaint of "diagnosed Monday with mumps" was identified during a regular local review of syndromic surveillance data. Local public health follow up with the infection preventionist revealed that the child was fully vaccinated and had no known exposure to mumps. The patient was brought to the emergency department due to concerns that he was misdiagnosed at the clinic where he was seen previously. Unfortunately, the patient was seen outside of the optimal window for testing, but the evaluation at the emergency department and additional follow up by public health made the concern for mumps very low.

Soon after that follow up was initiated, the local health department received a call from the general public about a sign put up at local recreational center stating that children attending the center had been exposed to mumps. There were no known cases of mumps in Tennessee at that time and the only active mumps investigation in the local jurisdiction was the follow up on the emergency department visit. Rapid follow up with the parent of the patient confirmed that they were the "mumps" exposure being referred to at the recreational center and public health was able to work with the recreational center to remove the signage, as the risk of the patient actually having mumps was low. Information from the investigation initiated by the syndromic surveillance data allowed for public health to rapidly connect the dots between the exposure notice and the patient.

\section{Conclusions}

Regular monitoring of syndromic surveillance data provides important opportunities for public health intervention that would not be possible otherwise. In all of the instances mentioned, timely syndromic surveillance data monitoring and follow up benefited public health responses by filling information gaps, helping initiate conversations with hospitals, and serving as another safety net for unreported illnesses. Conditions with post-exposure prophylactic interventions that can benefit from increased lead time are valuable targets for routine syndromic surveillance.

ISDS Annual Conference Proceedings 2019. This is an Open Access article distributed under the terms of the Creative Commons AttributionNoncommercial 3.0 Unported License (http://creativecommons.org/licenses/by-nc/3.0/), permitting all non-commercial use, distribution, and reproduction in any medium, provided the original work is properly cited. 